\title{
Conservation of wild silkworm genetic resources through cryopreservation: Standardization of sperm processing
}

\section{G. Lokesh*}

Central Sericultural Germplasm Resources Centre, Central Silk Board, Hosur - 635109

(Tamil Nadu), India

Geetha N Murthy

Central Sericultural Germplasm Resources Centre, Central Silk Board, Hosur - 635109 (Tamil Nadu), India

\section{Veeranna Gowda}

Central Sericultural Germplasm Resources Centre, Central Silk Board, Hosur - 635109

(Tamil Nadu), India

\section{Alok Sahay}

Central Sericultural Germplasm Resources Centre, Central Silk Board, Hosur - 635109

(Tamil Nadu), India

Gargi

Central Sericultural Germplasm Resources Centre, Central Silk Board, Hosur - 635109 (Tamil Nadu), India

${ }^{*}$ Corresponding author. E-mail: lokesh10csb@gmail.com

\section{Abstract}

Conservation of the invaluable sericigenous genetic resources is of prime importance with respect to their utilization and improvement for wider exploitation. Conservation of wild silkworms and its applicability in hybridization have limitations due to incompatibility, less amenability, change of behaviour under ex situ conditions, non-synchronization of moth eclosion and difficulties in mating between variables. In view of this, the newer technologies such as cryopreservation and artificial insemination are offering better strategies for preservation of biologically active samples like semen at sub-zero temperature $\left(-196^{\circ} \mathrm{C}\right)$ conditions for longer duration. In this context, under standardization of sperms preservation from wild silkworms, two methods of semen collection were scrutinized for obtaining active and viable sperm for cryopreservation and further artificial insemination. Semen collection from the seminal vesicle of freshly emerged male moth and the other from the bursa copulatrix (BC) and spermatheca of the female moth after mating. The sperms in the semen collected from seminal vesicle are in the form of bundles known as eupyrene sperm bundles and apyrene sperms. The morphology and behaviour of these sperm bundles were recorded through microscopic examination. To study the density and motility behaviour of the sperms, sperm bundles were treated with proteolytic enzyme $(\sim 2-3 \mu \mathrm{g} / \mathrm{ml})$ to digest the membrane and release the sperms. The density and motility behaviour of sperms in the semen recovered from the BC and spermatheca of female moth after mating were higher compared to those released after digestion of sperm bundles from seminal vesicle of the male moth.

Keywords: Conservation, Cryopreservation, Seminal vesicle (SV), Silkworm, sperm, Sperm motility

\section{INTRODUCTION}

Vanya silks are the source of natural silk fibres produced from wild silkworms such as Tasar, Muga and Eri. These wild silkworm fauna are unique with vast genetic resources in India, playing a crucial role in the development of sericultural industry in the country. It is reported that the family Saturniidae comprises of about 1200 1500 species all over the world of which the Indian sub-continent, extending from Himalayas to Sri Lanka may possess over 50 species (Nassig et al.

\section{Article Info}

DOI:10.31018/jans.v10i2.1733

Received: November 29, 2017

Revised: January 19, 2018

Accepted: March 19, 2018

How to Cite

Lokesh, G. et al. (2018). Conservation of wild silkworm genetic resources through cryopreservation: Standardization of sperm processing. Journal of Applied and Natural Science, 10(2): 544 - 547 
situ methods. Conservation of domesticated silkworms is well standardized and the generations are maintained by rearing under controlled environmental conditions at many agro-climatic regions of the country (Dayananda et al., 2011). Acclimatization and adaptation of domesticated silkworms to new environment takes place without much change in their behaviour and productivity. They are maximum utilized as evidenced by increased production and improvement through hybridization based on selection for desired traits among the diverse gene pool(Lakshmi et al. , 2011). On the contrary, conservation of wild silkworms particularly the tasar silkworm Antheraea mylitta and its applicability in hybridization have limitations due to incompatibility, less amenability to human handling, change of behaviour as well as product quality under ex situ conditions, nonsynchronization of moth emergence and difficulties in mating between different ecoraces (Lokesh et al., 2015).

The advent of newer technologies such as artificial insemination and cryo-preservation of sperm offer better strategies for preservation of biologically active samples like semen at sub-zero temperature conditions $\left(-196^{\circ} \mathrm{C}\right)$ for longer duration (Banno et al., 2013; Sahara et al., 2003; Takemura et al.,2000). Takemura et al., 1996 demonstrated that virgin females artificially inseminated with seminal fluid collected from seminal vesicle and mated females could oviposit fertilized eggs at almost similar rates. An endopeptidase of serine protease type in the glandula prostatica of B.mori is reported important for acquisition of sperm motility during passage through seminal vesicle to the ejaculatory duct (Osanai and Isono 1997). Artificial insemination in $B$. mori using trypsin treated sperm and without secretion of glandula prostatica could yield fertilized eggs (Takemura et al., (1999). Thus, artificial insemination technique would be very useful during physiological studies on insect fertilization and reproduction. This technique could also be helpful to trace transfer of sperm from bursa copulatrix to seminal receptacle in the female reproductive tract right from insemination in real-time (Takemura et al, 2006). Silkworm sperms are usually dimorphic (Yamashiki, and Kawamura, 1997; Banno et al., 2013) wherein the functional eupyrene sperms have an extended glycocalyx with blade-like structures called laciniate appendages, while, apyrene sperm is a parasperm lacking nucleus and appendages (Hamon and Chauvin, 1992). Although several studies have been taken up on artificial insemination in B.mori, it is very limited in wild silkworms like Antheraea mylitta (Ravikumar et al., 1998). The standardization and utilization of artificial insemination techniques would aid in effective conservation of invaluable tasar silkworm genetic resources. In this context, a study was attempted to develop/standardize the protocol for collection of semen from male moth of A.mylitta, processing of semen for cryopreservation and artificial insemination which would go a long way in solving reproductive problems as well as for selective breeding based on desired traits.

\section{MATERIALS AND METHODS}

Semi-domesticated tropical tasar silkworm A.mylitta cocoons of bivoltine race were collected from Central Tasar Research \& Training Institute, Ranchi during first and second grainage seasons i.e., July and September 2016. After emergence, the male and female moths were collected separately. Some of the moths were allowed to mate and few were used for dissection to collect semen.

Dissection and collection of semen: Male tasar moth (Fig.1a) was used for collection of sperm from seminal vesicle by dissecting in Ringer's saline solution. The seminal vesicle (SV) was separated from the reproductive system (Fig.1b) and collected in a micro centrifuge tube containing Grace's insect culture medium (GICM- Amino acids, Vitamins, Sugars with L-glutamine and without sodium bicarbonate ) and gently tapped to release the seminal fluid. The seminal fluid obtained was centrifuged at $3000 \mathrm{rpm}$ for $3 \mathrm{~min}$. and the collected supernatant was observed under microscope.(300x) The supernatant was kept in a sterilized ice cold microtube until use for further process.

Recovery of semen from female moth: After mating for $1 \mathrm{hr}$., the female moths were decoupled and collected for dissection. The bursa copulatrix (BC) and spermatheca were carefully dissected out and placed in a $1 \mathrm{ml}$ tube, the membrane was ruptured and contents collected. The BC fluid was subjected to microscopic study.

Semen processing with protease enzyme: The trypsin solution was prepared in cell culture medium (GISM). The seminal fluid recovered from SV was separately mixed with different concentrations of trypsin solution i.e., $0.5 \mu \mathrm{g} / \mathrm{ml}, 01 \mu \mathrm{g} / \mathrm{ml}$, $02 \mu \mathrm{g} / \mathrm{ml}, 3 \mu \mathrm{g} / \mathrm{ml}, 4 \mu \mathrm{g} / \mathrm{ml}$ and $5 \mu \mathrm{g} / \mathrm{ml}$. The mixture was then incubated at $25^{\circ} \mathrm{C}$ for $15 \mathrm{~min}$ to digest the spermatophore membrane and release sperms. The efficiency of trypsin activity was examined through microscopic observations (300x) separately for different concentrations (Takemura, 1999).

Dilution of semen in Ringer's saline solution: Since there was clumping of sperm cell in the medium and in order to standardize the dilution of semen sample for microscopic examination, three different dilutions of the samples of semen obtained from SV and BC were prepared using GISM i.e., $50 \%, 30 \%, 20 \%, 10 \%$ and $5 \%$. The samples from each dilution were observed under the microscope and the sperm activity was recorded. 


\section{RESULTS AND DISCUSSION}

The microscopic examination of the seminal fluid collected from SV of adult male reproductive system revealed pleomorphic bundle like structures. Large and longer bundles were identified as eupyrene sperm bundles which are less in number and smaller bundles which are more in numbers as apyrene bundles. Nil or hardly any movement of these bundles was observed (Fig.2). Similar observations have been earlier reported in A.mylitta (Ravikumar et al., 1998) and B.mori (Yamashiki and Kawamura, 1997; Kawamura et al., 2000). Two types of sperms i.e., eupyrene and apyrene are found in the seminal vesicle of male reproductive organ. Both the sperm types are inactive and remain in bundles until they reach the female reproductive system through bursa copulatrix during the process of copulation (Ravikumar et al., 1998). In both types, 256 sperm cells are surrounded by a layer of cyst cells after maturation division, later forming a sperm bundle. The marked differences between the two are: 1) the eupyrene sperm is an ordinary sperm with a spearhead-shaped nucleus anteriorly, while, the apyrene sperm loses its nucleus at a very late stage of spermatogenesis; 2) the length of the apyrene sperm is half that of the eupyrene sperm; 3 ) the centriole/basal body behaves quite differently (Yamashiki and Kawamura, 1997) and 4) the two types differentiate during distinctly different phases of metamorphosis, the eupyrene sperm in the larval stage and the apyrene sperm after spin-

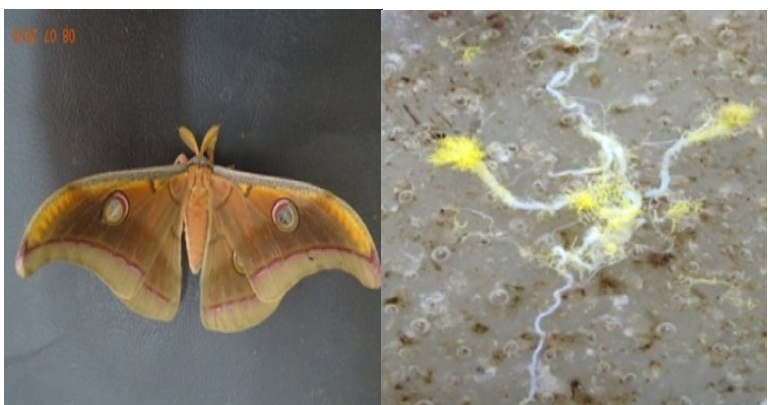

Fig. 1. a. Male A.myltta moth., b. Male reproductive system of A.myltta.

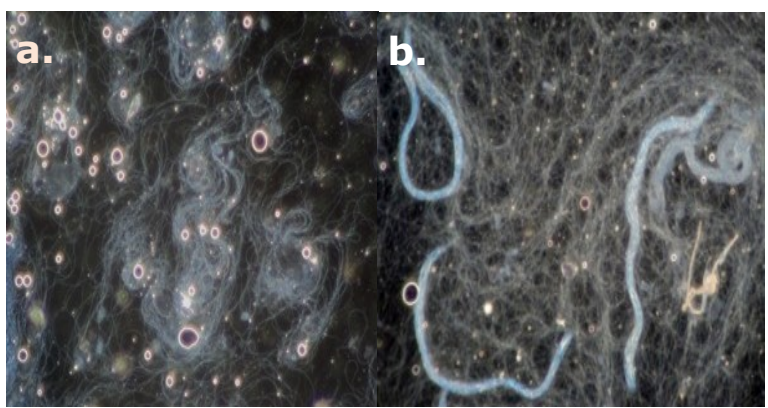

Fig. 2. a. Microscopic observations (300X) of Seminal fluid b. SV fluid consisting Eupyrene sperm bundles and apyrene sperms. ning. (Kawamura et al., 2000; Fugo et al., 1996). The bursa copulatrx (BC) of the female silkmoth is filled with various secretions and during mating the seminal fluid is transferred from the male reproductive system. The contents are all heterogeneous, since they are formed by the partially mixed, viscous streams of the male secretions. The microscopic analysis of $\mathrm{BC}$ content of adult female $A$. mylitta revealed high viscosity with dense spermatozoa. The spermatozoa were active and showing vigorous motility in different forms. The apyrene movement was observed for a short while, whereas, eupyrene movement was constant for a longer period. Similar observations were reported by Ravikumar et al.(1998).

Semen samples collected from the SV were subjected to trypsin treatment to dissolve the sperm bundles and facilitate sperm activation. Various concentrations of trypsin were examined for identifying effective optimum concentration of trypsin for sperm activation and membrane dissolution. It was observed that, higher concentration of trypsin $>3 \mu \mathrm{g} / \mathrm{ml}$ affected the digestibility as well as sperm activity. After treatment with higher concentration of trypsin, there was no appearance of either sperm bundles or active spermatocytes (Fig.4a).We could only observe dispersed inactive spermatocytes. At lower concentration of $1 \mu \mathrm{g} / \mathrm{ml}$, there was no effective dissolution of the bundles (Fig.4b). However the trypsin concentration at $2 \mu \mathrm{g} /$ $\mathrm{ml}$ to $3 \mu \mathrm{g} / \mathrm{ml}$ revealed free and active spermatocytes. Takemura (1999) reported that when

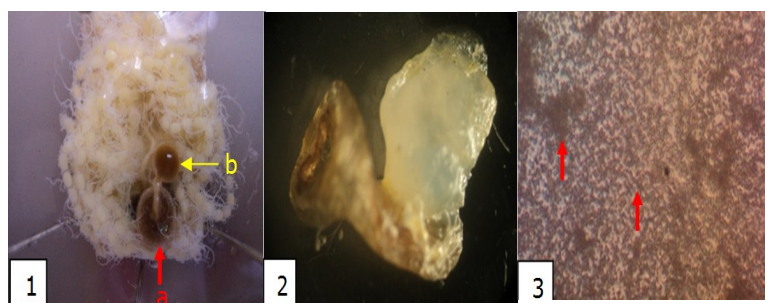

Fig. 3. Bursa copulatrix of female adult: 1. Dissected reproductive system of female moth (a. bursa copulatrix, b. Spermatheca). 2. Section of Bursa copulatrix.3. Content of BC showing clump formation of spermatocytes and motile spermatocytes (200X).

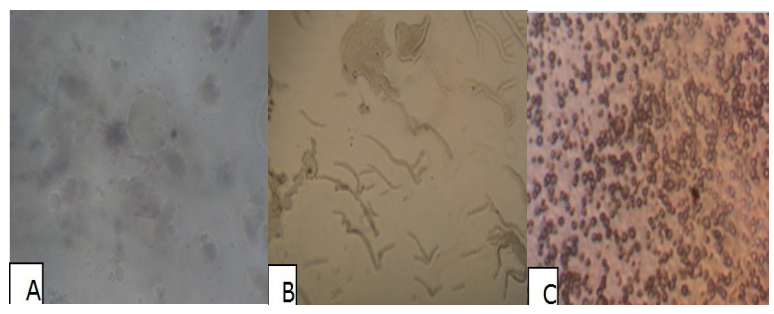

Fig. 4. Semen treated with various concentrations of trypsin (600X): $\boldsymbol{A}$ - high concentration $(>3 \mu \mathrm{g} / \mathrm{ml})$ of trypsin; B - Low concentration of trypisin $(<1 \mu \mathrm{g})$ $\mathrm{ml})$ treated semen; $\mathbf{C}$ - Semen treated with 2 to $3 \mu \mathrm{g} / \mathrm{ml}$ trypsin. 


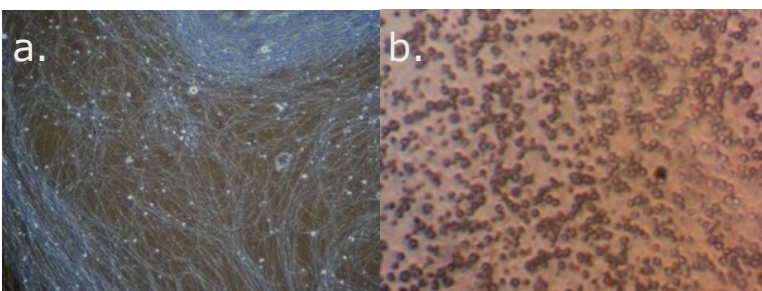

Fig. 5. a. Trypsin treated semen sample collected from SV $\boldsymbol{b}$. Semen sample collected from BC after $1 \mathrm{hr}$. of mating.

sperms were incubated without trypsin, eupyrene sperm remained in bundle form and apyrene sperm moved slowly. On the other hand, when the sperms were treated with $0.3 \mu \mathrm{g} / \mathrm{ml}$ concentration of trypsin, eupyrene sperm bundles completely dissociated, like those collected from the BC of normally mated females. Further, the optimal dilution of the semen sample with the Insect Ringers' solution was examined that could facilitate microscopic analysis and to study the spermatocyte density/count in the semen sample. Various dilutions of semen and subsequent analysis revealed that $10 \%$ dilution $\left(10^{-1}\right)$ at room temperature $(25 \pm$ $2^{\circ} \mathrm{C}$ ) is suitable for the study of sperm density and also to evade clumping of spermatocytes during microscopic analysis. Observations of semen sample from both SV and BC at $10^{-1}$ dilution showed a comparatively higher density of spematocytes in the BC semen sample. To be certain of obtaining reproducible data, the sample should be thoroughly diluted before aliquots are taken for assessment.

\section{Conclusion}

It is highly pertinent to understand and to standardize the protocols for the collection of semen and processing the semen to get viable active sperms which are appropriate for insemination in virgin female moths of wild sericigenous insects particularly in the tropical tasar silkworms. Based on the present observations, it was recorded that the successful activation of the eupyrene sperm bundles using standardized dose of trypsin at the concentration $2 \mu \mathrm{g} / \mathrm{ml}$ to $3 \mu \mathrm{g} / \mathrm{ml}$ is promising in standardization of other processing of A.mylitta sperms to get viable semen for subsequent successful artificial insemination. Furthermore, this technique of artificial insemination and semen cryopreservation paves way for its application in long-term preservation of wild seri-genetic resources using cryopreserved sperms in the future.

\section{REFERENCES}

Banno Y., Kiyomi Nagasaki, Marino Tsukada, Yuko Minohara, Junko Banno, Kazuhiro Nishikawa, Kazunori Yamamoto, Kei Tamura, Tsuguru Fujii. (2013). Development of a method for long-term preservation of Bombyx mori silkworm strains using frozen ova- ries. Cryobiology. 66:283-287.

Chinnaswamy, K.P. (2001). Sericulture biodiversity in India. pp. 54-61. In: M.K. Balla, S. Rayamajhi and N.M.B. Pradhan (eds.) Participatory Biodiversity Conservation in South Asia Region. Fonarem, Kathmandu, Nepal.

Dayananda, Kulkarni, S.B., Ramamohan Rao,P., Gopinath, O.K. and Nirmal Kumar (2011). Evaluation and selection of superior bivoltine hybrids of the silkworm Bombyx mori L. For tropics through large scale In-House testing. International Journal of Plant, Animal and Environmental Sciences. 1(3):16-22.

Fugo, H., Yamauchi, M. and Dedos, S. G. (1996). Testicular ecdysteroids in the silkmoth, Bombyx mori. Proc. Jpn. Acad. 72:34-37.

Hamon, C. and Chauvin, G. (1992). Ultrastructural analysis of spermatozoa of Korscheltellus lupinus L. (Lepidoptera, Hepialidae) and Micropterix calthella L. (Lepidoptera, Micropterigidae). Int. J. Insect Morphol. Embryol. 21:149-60.

Kawamura Naoko, Naoko Yamashiki,Hiroshi Saitoh,and Ken Sahara. (2000). Peristaltic Squeezing of Sperm Bundles at the Late Stage of Spermatogenesis in the Silkworm, Bombyx mori. Journal of Morphology 246:53-58.

Lakshmi, H., Chandrashekharaiah, Ramesh Babu, M., Raju, P.J., Saha, A.K. and Bajpai, A.K. (2011). HTO5 x HTP5, The new bivoltine silkworm (Bombyx mori L.) hybrid with thermo-tolerance for tropical areas. International Journal of Plant, Animal and Environmental Sciences. 1(2):88-104.

Lokesh, G., Srivastava, A.K., Srivastava, P.P., Kar, P.K. and Alok Sahay (2015). Conservation and breeding strategies for Laria silkworm, Antheraea mylitta Drury for exploitation of Sal flora. Sericologia. 55(3):151158.

Nassig, W.A., Lempe,R.E.J. \&and Kger, S. (1996). The Saturniidae of Sumatra (Lepidoptera). Heterocera Sumatrana 10: 3-10.

Osanai, M., Isono, M., (1997). Dissociation of eupyrene sperm bundle by acids, especially succinate accumulated in the spermatophore of the silkmoth, Bombyx mori. Invertebrate Reproduction and Development 31: 99-108.

Ravikumar G., Rajeswary,H., OjhaN.G.and Sinha S.S. (1998). Sperm transfer and sperm activation in Tasar silkmoth Antheraea mylitta. Korean.J.Seric.Sci. 40(1): 33-37.

Sahara, K., Takemura,Y. (2003) Application of artificial insemination technique to eupyrene and/or apyrene sperm in Bombyx mori , J. Exp. Zool. 297: 196- 200.

Takemura, Y., Kanda,T., Tamura,T. Shinbo, H. and Horie, Y. (1996). Development of new method for artificial insemination of the silkworm, Bombyx mori. J. Seric. Sci. Jpn. 65: 456-463.

Takemura Y, Kanda,T. And Horie,Y. (1999). Artificial insemination using trypsin-treated sperm in the silkworm, Bombyx mori , J. Insect Physiol. 45: 471-477.

Takemura Y., Kanda, T. And Horie,Y. (2000). Artificial insemination using cryopreserved sperm in the silkworm, Bombyx mori , J. Insect Physiol. $46: 491-497$.

Takemura,Y., Sahara,K. Mochida, Y. and Ohnuma, A. (2006). Apyrene sperm from the triploid donors restore fecundity of cryopreserved semen in Bombyx mori. J. Insect Physiol. 52: 1021-1026.

Yamashiki, N. and Kawamura, N. (1997). Behaviors of nucleus, basal bodies and microtubules during eupyrene and apyrene spermiogenesis in the silkworm, Bombyx mori (Lepidoptera). Dev. Growth Differ 39:715-722. 